\title{
A continuous scanning mode for ptychography
}

\author{
Jesse N. Clark, ${ }^{1, *}$ Xiaojing Huang, ${ }^{2}$ Ross J. Harder, ${ }^{3}$ and lan K. Robinson ${ }^{4,5}$ \\ ${ }^{1}$ London Centre for Nanotechnology, University College London, London WC1E 6BT, UK ${ }^{\dagger}$ \\ ${ }^{2}$ National Synchrotron Light Source II, Brookhaven National Laboratory, Upton, NY 11973, USA \\ ${ }^{3}$ Advanced Photon Source, Argonne National Laboratory, Argonne, IL 60439,USA \\ ${ }^{4}$ London Centre for Nanotechnology, University College London, London WC1E 6BT, UK \\ ${ }^{5}$ Research Complex at Harwell, Didcot, Oxfordshire OX11 ODE, UK
}

compiled: September 17, 2014

\begin{abstract}
We outline how ptychographic imaging can be performed without the need for discrete scan positions. Through an idealized experiment, we demonstrate how a discrete position scan regime can be replaced with a continuously scanned one with suitable modification of the reconstruction scheme based on coherent modes. The impact of this is that acquisition times can be reduced, significantly aiding ptychographic imaging with X-rays, electrons or visible light.

OCIS codes: (100.5070) Image processing : Phase retrieval; (180.5810) Microscopy : Scanning microscopy; (180.7460) Microscopy : X-ray microscopy

http://dx.doi.org/10.1364/XX.99.099999
\end{abstract}

Ptychography [1] is a microscopy technique that permits high resolution imaging with X-rays [2, 3], electrons $[4,5]$ and visible light [6] in 2- and 3-dimensions [7] on biological [8] and materials science samples [9]. Ptychography works by collecting a series of $J$ diffraction measurements from a sample, $O$, that is rastered through an illumination or probe, $P$, at discrete points $j$ with adjacent positions sharing some overlap. Due to large redundancy in the data sets, both the probe $[3,10]$ and object can be reconstructed simultaneously using phase retrieval. Recent algorithmic advances have extended the technique to reconstruct multiple probe and object modes [11], for example when the illumination is partially coherent, the sample is dynamic [12] or there is significant detector point spread [13]. Additionally the algorithms can be modified to account for multiple scattering from thick specimens [14] or when the data are under sampled on the detector [15].

The experimental setup of ptychography shares a number of similarities with scanning probe techniques such as scanning transmission x-ray microscopy (STXM) and scanning electron microscopy (SEM) in that a probe is scanned across the sample with the transmitted (or diffracted) wave field intensity recorded. However, for rapid data acquisition, the techniques such as STXM or

\footnotetext{
* Corresponding author: jesclark@stanford.edu.

† Stanford PULSE Institute, SLAC National Accelerator Laboratory, 2575, Sand Hill Rd, Menlo Park and Center for Free-Electron Laser Science (CFEL), Deutsches Elektronensynchrotron (DESY), Notkestrasse 85, 22607 Hamburg, Germany.
}

SEM can acquire data while the sample (or probe) is scanned continuously across the sample without collecting data at discrete positions. This has the benefit that collection times can be reduced since no time is wasted waiting for motors to move and settle. Current state of the art setups still require motor settling times $(0.15 \mathrm{~s})$ that are similar to the exposure time $(0.2 \mathrm{~s})$ [16] for each position. Due to the large number of diffraction patterns collected for ptychography (typically thousands), a reduction in acquisition time would be highly desirable. In this letter we demonstrate that a continuous scan regime (or 'fly scan') is equivalent to a degradation in spatial coherence. We approximate the continuous scanning by summing diffraction from adjacent positions and show that the deleterious effects of this can be ameliorated through post-processing. The reduction in collection time using this scheme should enable studies of dynamics and in situ processes as well as reducing stability requirements on experimental components.

For a quasi-monochromatic, spatially coherent probe, the intensity at a position $j$ will take a form of;

$$
I_{j}(\boldsymbol{q})=\left|\mathcal{P}_{z}\left[P(\boldsymbol{r}) O\left(\boldsymbol{r}+\boldsymbol{r}_{j}\right)\right]\right|^{2},
$$

where $\boldsymbol{r}$ is a real-space position coordinate, $\boldsymbol{r}_{j}$ is the translation of the object (Fig. 1 (a)), $\boldsymbol{q}$ is a reciprocal space position coordinate and $\mathcal{P}_{z}$ is the Fresnel freespace propagator for a distance $z$ [17]. If we consider the case of a continuously scanned sample with a detector that has finite detection time $T$, then the recorded intensity will now contain additional contributions coming from the positions around the previously discrete scan 
positions. The intensity can now be written as;

$$
I_{j}(\boldsymbol{q})=\int_{t_{1}}^{t_{2}}\left|\mathcal{P}_{z}\left[P(\boldsymbol{r}) O\left(\boldsymbol{r}+\boldsymbol{r}_{j}+\boldsymbol{v} t\right)\right]\right|^{2} \mathrm{~d} t
$$

where $t$ is time, $\boldsymbol{v}$ is a scan velocity (identical, but not necessarily constant for all $j$ positions) and $T=t_{2}-t_{1}$ is the (constant) integration time (Fig. 1 (b)). We now select a sufficiently small time step $\Delta t$, so that we can discretize Eq. 2 and sum over $N$ discrete time steps to get,

$$
I_{j}(\boldsymbol{q})=\sum_{n=0}^{N-1} \Delta t\left|\mathcal{P}_{z}\left[P(\boldsymbol{r}) O\left(\boldsymbol{r}+\boldsymbol{r}_{j}+\boldsymbol{v} n \Delta t\right)\right]\right|^{2} .
$$

Since only relative translations are relevant, the translation $\boldsymbol{r}_{n}=\boldsymbol{v} n \Delta t$ can be absorbed into the probe modes giving;

$$
I_{j}(\boldsymbol{q})=\sum_{n=0}^{N-1} \Delta t\left|\mathcal{P}_{z}\left[P\left(\boldsymbol{r}-\boldsymbol{r}_{n}\right) O\left(\boldsymbol{r}+\boldsymbol{r}_{j}\right)\right]\right|^{2} .
$$

which states that the intensity is now an incoherent sum of intensities from translated probes (probe modes). The significance of this is that a data set taken without discrete position data (continuous scanning) can be recast as a periodic sample vibration problem (Eq. 3 ) with sample translations of $\boldsymbol{r}_{n}$, as illustrated in Fig. 1. The case of a vibrating sample has been shown to be equivalent to a degradation in spatial coherence [18] and reconstructible [12](for both discrete and continuous motion) using recent algorithmic advances [11] by absorbing the sample motion into the probe modes (Eq. 4). For constant velocity, the integration time of the detector will dictate the apparent degree of partial coherence. This observation leads us to a simple heuristic for the scaling between the apparent coherence length, $\sigma_{l}$, and scanning, via $\sigma_{l} \propto(|\boldsymbol{v}| T)^{-1}$. It should be noted that the $N$ illumination modes appearing in Eq. 4 can be written as an orthogonal set of $N^{\prime}$ modes (with $N^{\prime} \leq N$ ) that are themselves fully coherent but mutually incoherent [19]. The implications of this, along with the assertion that the integration time of the detector determines the apparent coherence length, means that the number of relevant modes $N^{\prime}$ does not scale with our arbitrarily assigned number $N$, but instead scales with $\sigma_{l}$. This can be seen in Fig. 1 (d), which shows the mode power for the first 5 orthogonal modes $\left(n^{\prime}\right.$, obtained using singular value decomposition) calculated using different numbers of simulated horizontally translated probes $(N)$ (but with the same maximum translation, i.e. coherence length). The mode power is relatively insensitive to the number of translated probes but instead depends primarily on the maximum translation. The probe function used here was a Gaussian with maximum translation of one probe width (full-width at half maximum).

To test the ability of operating ptychography in a continuous scan regime, experiments were carried out at (a)

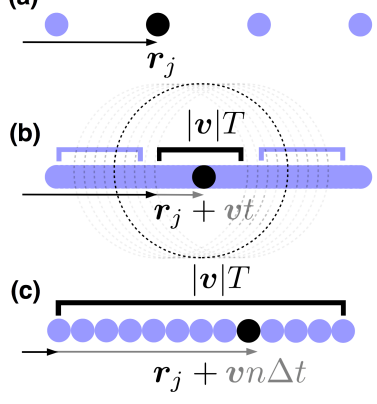

(d)

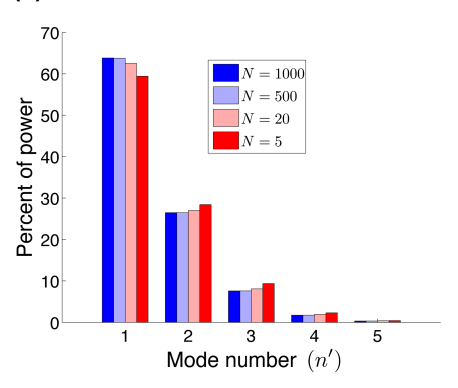

Fig. 1. (a) Conventional ptychography uses data collected with discrete translations $\boldsymbol{r}_{j}$. (b) For a continuously scanned sample with constant velocity $\boldsymbol{v}$, the diffraction data will be made up of the positions that occupy a length $|\boldsymbol{v}| T$ around the scan positions $\boldsymbol{r}_{j}$ due to an integration time of $T$. (c) The continuous scan can be discretized with steps separated by a small distance $\boldsymbol{v} \Delta t$. (d) Mode power for the first 5 orthogonal modes $\left(n^{\prime}\right)$ calculated using different numbers $(N)$ of simulated translated probes, but with the same maximum translation $\left(\sigma_{l}\right)$. The number of relevant modes is relatively insensitive to the number of translated positions but depends on the apparent coherence length.

beamline 34 ID-C at the Advanced Photon Source in Chicago. A Kirk-Patrick Baez (KB) mirror system [20] was used to focus $9 \mathrm{keV} \mathrm{X}$-rays onto a sample which was placed at the approximate focus, $100 \mathrm{~mm}(200 \mathrm{~mm})$ from the vertical (horizontal) mirror. Horizontal and vertical slits prior to the KB mirrors were used to adjust the spatial coherence entering the optics and resulted in a probe of $700 \mathrm{~nm}$ (horizontally) by $1000 \mathrm{~nm}$ (vertically) at the sample position. The sample was a lithographed 1.5 $\mu \mathrm{m}$ thick tungsten test pattern. Scanning of the sample stage perpendicular to the X-ray direction was achieved by a nPoint piezo scanner NPXY100Z25. Diffraction was recorded $2 \mathrm{~m}$ downstream using a TimePix pixel detector [21], consisting of $256 \times 256$ pixels of side length $55 \mu \mathrm{m}$, which allows detection of individual photons. At each position, 5 exposures of $0.5 \mathrm{~s}$ duration were collected. To improve dynamic range of the data, a partially attenuating beamstop [22] was placed over the central region of the diffraction pattern. The beamstop consisted of a $200 \mu \mathrm{m}$ thick silicon square which covered $30 \times 30$ pixels. The central region of the beamstop was scaled by the calculated absorption using tabulated values [23]. To reduce absorption from air between the sample and detector, a $1 \mathrm{~m}$ evacuated flight tube was installed (with Kapton windows).

A grid scan of 81 points horizontally (with $100 \mathrm{~nm}$ step size) and 11 points vertically (with $200 \mathrm{~nm}$ step size) was performed on a region of the sample that contained numerals and bars. Both the probe and object were reconstructed simultaneously using a multi-resolution approach where initial low-resolution reconstructions were obtained (using cropped data) which were then used to seed the next higher-resolution reconstruction $[12,24]$. Three levels of resolution were used $(64 \times 64,96 \times 96$, and 
$128 \times 128$ pixels), each lasting 500 iterations where propagation was achieved using a fast Fourier transform. The object was initialized with an array of random numbers and the probe from the KB mirror system was modeled as a rectangular lens. Position correction [10] was used for the highest resolution only, implemented using a local search (restricted to the 9 closest pixels) and selecting the position which minimized the difference between the measured and calculated diffraction.

Shown in Fig. 2 (a) is a reconstruction using all grid positions and a single sample and probe mode which amounts to a regular ptychography experiment (assuming full coherence) with intensity model given by Eq. 1. The sample features are well reproduced, with bars clearly visible along with some numerals at a resolution of $40 \mathrm{~nm}$ as determined from the phase retrieval transfer function [25]. To test the equivalence of a continuously scanned sample and partial coherence, a new data set was created by summing intensity measurements (in the horizontal direction only) from adjacent positions into bins to form a new set of diffraction measurements (Fig. 1 (c)). Two new data sets were created by summing diffraction from 4 and 7 adjacent horizontal positions (resulting in an intensity model of Eq. 3). The new data sets created from the 4 and 7 position summing now contained 20 and 11 points in the horizontal direction with step sizes of $400 \mathrm{~nm}$ and $700 \mathrm{~nm}$ respectively. The last columns of the scan in the horizontal direction were omitted appropriately to obtain an integer number of points in the new scan. The increasing number of positions is equivalent to an increasing integration time or scan speed.

Figures 2 (b)-(c) shows reconstructions from the new data sets using a single illumination mode for the summing of 4 and 7 positions respectively. There is a clear degradation in the image quality (compared to Fig. 2 (a)) with some distortion of the bars and numbers and an increased non-uniformity in the feature free regions. This failure is attributed to the fact using a single illumination mode does not adequately describe the forward process given by Eq. 3. With increased summing we see a degradation in the pertinent features of the image with the numbers becoming very hard to identify. The increased degradation with the size of the summation can be understood through the equivalence of the summing with partial spatial coherence, i.e. the larger the distance the summing occurs over, the smaller the (apparent) coherence length. To ameliorate the deleterious effects introduced by the summing, a second reconstruction was performed on the original and summed data sets using 5 illumination modes (initiated using a previous method [12]). The reconstructions for the data sets are shown in Figs. 2 (a)-(d) (right hand column, 5 modes) and the probes in Fig. 3. The reconstruction now reproduces the relevant features well, with the bars and numerals easily recognizable and represent a significant improvement over the single mode reconstructions, supporting the thesis that continuous scanning can be

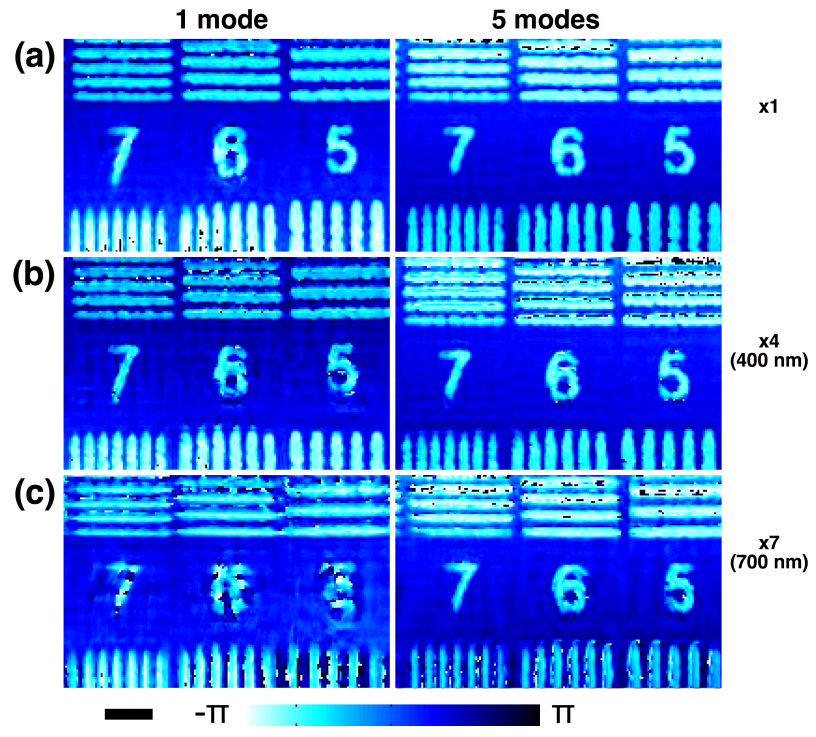

Fig. 2. (a) Reconstructed object phase assuming full coherence ( 1 mode,left) and partial coherence ( 5 modes, right) for the original data. (b) Reconstruction using new positions that consist of summing 4 adjacent positions (over $400 \mathrm{~nm}$ horizontally), approximating a continuously scanned sample. The reconstruction assuming full coherence (left) is degraded compared to (a) but shows improvement when assuming partial coherence (right). This is also shown for a larger ((d), 7 positions, $700 \mathrm{~nm}$ ) level of summing which would be equivalent to an increased scanning speed. With the increasing number of positions the reconstructions degrade (left) when the apparent reduction in spatial coherence is not taken into account. The scale bar is $1 \mu \mathrm{m}$.

modeled as partial spatial coherence. The original reconstruction (Fig. 2 (a)) has seen some improvement, indicating that the illuminating wave field was not quite fully coherent. Figure 3 shows the relative orthogonal probe mode power from the reconstructions. It can be seen that the increased summing has resulted in a commensurate decrease in the primary mode power and increase in higher $\left(n^{\prime} \geq 2\right)$ order mode powers, a clear indication of partial coherence $[11-13,19,26]$. An outstanding question is how many modes are required and how should this number be determined? Although a clear answer remains elusive and beyond the scope of this work, one strategy that could be employed would be to gradually incorporate more modes into the iterative procedure until the power in the highest (orthogonal) mode falls below some threshold value, for example $1 \%$.

Finally, we note that there are still some small scale artifacts present in the reconstructions approximating the continuous scans. Possible explanations could be that there has been an apparent increase in step size, reducing the average overlap [12] from 0.92 for the original data set 0.56 for the 7 summed position data set. However, a more likely explanation comes from noting that there is a requirement that the velocity be identical (but not necessarily constant) around each scan position (Eq. 2). 
In reality, any positional errors that are present before summing and creating the new data sets will cause the effective velocity to be different for every scan position, violating the previously stated requirement. By comparing the original positions with those after iterative refinement for the original data without summation, we found that the average error for the horizontal positions was $3 \%$ and for the vertical, $12 \%$. The actual positions (particularly the vertical), have enough error that when summing the data over adjacent positions that the relative translations for each summed region are no longer identical, meaning Eq. 4 is not entirely true, even if position refinement is performed on the summed data.

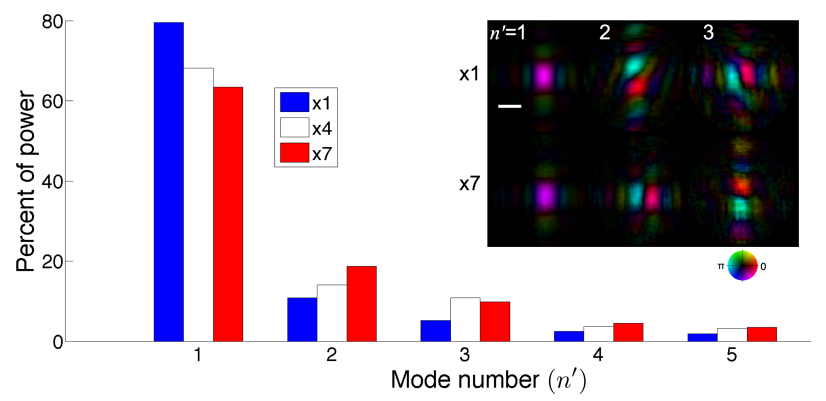

Fig. 3. An increase in position summing $(1 \rightarrow 7)$ results in a decrease in the power contained in the primary mode $\left(n^{\prime}=1\right)$ while the higher order modes $\left(n^{\prime} \geq 2\right)$ show a commensurate increase in relative power. The three modes with the most power are shown for the original data set and the $\times 7$ summing. The scale bar is $1 \mu \mathrm{m}$.

We have demonstrated the equivalence of a continuous scanning regime in ptychography with partial spatial coherence. This scanning regime eliminates the need for discrete positions, avoiding time overheads associated with moving the object or probe. We anticipate this scheme will be adopted for rapid data collection across different ptychographic imaging modalities using x-rays, electrons and visible light, enabling a plethora of new and exciting science.

This work was supported by an FP7 advanced grant from the European Research Council from the EPSRC under grant no. EP/I022562/1. X. H. is supported by the Department of Energy, Office of Basic Energy Sciences under Contract No. DE-AC-02- 98CH10886. The experimental work was carried out at the Advanced Photon Source Beamline 34-ID-C built with funds from the U.S. National Science Foundation under Grant No. DMR- 9724294 and operated by the U.S. Department of Energy, Office of Science, Office of Basic Energy Sciences under Contract No. DE-AC02-06CH11357. The authors gratefully acknowledge Graeme Morrison for design of the test pattern.

\section{References}

[1] R. Hegerl and W. Hoppe, Berichte Der BunsenGesellschaft Fur Physikalische Chemie 74, 1148 (1970).
[2] J. M. Rodenburg, A. C. Hurst, A. G. Cullis, B. R. Dobson, F. Pfeiffer, O. Bunk, C. David, K. Jefimovs, and I. Johnson, Phys. Rev. Lett. 98, 034801 (2007).

[3] P. Thibault, M. Dierolf, A. Menzel, O. Bunk, C. David, and F. Pfeiffer, Science 321, 379 (2008).

[4] C. T. Putkunz, A. J. D'Alfonso, A. J. Morgan, M. Weyland, C. Dwyer, L. Bourgeois, J. Etheridge, A. Roberts, R. E. Scholten, K. A. Nugent, and L. J. Allen, Phys. Rev. Lett. 108, 073901 (2012).

[5] M. J. Humphry, B. Kraus, A. C. Hurst, A. M. Maiden, and J. M. Rodenburg, Nat Commun 3, 730 (2012).

[6] J. M. Rodenburg, A. C. Hurst, and A. G. Cullis, Ultramicroscopy 107, 227 (2007).

[7] M. Dierolf, A. Menzel, P. Thibault, P. Schneider, C. M. Kewish, R. Wepf, O. Bunk, and F. Pfeiffer, Nature 467, 436 (2010).

[8] K. Giewekemeyer, P. Thibault, S. Kalbfleisch, A. Beerlink, C. M. Kewish, M. Dierolf, F. Pfeiffer, and T. Salditt, Proceedings of the National Academy of Sciences of the United States of America 107, 529 (2010).

[9] S. O. Hruszkewycz, M. V. Holt, C. E. Murray, J. Bruley, J. Holt, A. Tripathi, O. G. Shpyrko, I. McNulty, M. J. Highland, and P. H. Fuoss, Nano Letters 12, 5148 (2012).

[10] M. Guizar-Sicairos and J. R. Fienup, Opt. Express 16, 7264 (2008).

[11] P. Thibault and A. Menzel, Nature 494, 68 (2013).

[12] J. N. Clark, X. Huang, R. J. Harder, and I. K. Robinson, Phys. Rev. Lett. 112, 113901 (2014).

[13] B. Enders, M. Dierolf, P. Cloetens, M. Stockmar, F. Pfeiffer, and P. Thibault, Applied Physics Letters 104, 171104 (2014).

[14] A. M. Maiden, M. J. Humphry, and J. M. Rodenburg, J. Opt. Soc. Am. A 29, 1606 (2012).

[15] T. B. Edo, D. J. Batey, A. M. Maiden, C. Rau, U. Wagner, Z. D. Pešić, T. A. Waigh, and J. M. Rodenburg, Phys. Rev. A 87, 053850 (2013).

[16] M. Guizar-Sicairos, I. Johnson, A. Diaz, M. Holler, P. Karvinen, H.-C. Stadler, R. Dinapoli, O. Bunk, and A. Menzel, Opt. Express 22, 14859 (2014).

[17] D. M. Paganin, Coherent X-ray Optics (Oxford University Press, Oxford, 2006).

[18] J. N. Clark, C. T. Putkunz, E. Curwood, R. E. Scholten, I. McNulty, K. A. Nugent, and A. G. Peele, Optics letters 36, 1954 (2011).

[19] E. Wolf, J. Opt. Soc. Am. 72, 343 (1982).

[20] P. Kirkpatrick and A. V. Baez, J. Opt. Soc. Am. 38, 766 (1948).

[21] X. Llopart, R. Ballabriga, M. Campbell, L. Tlustos, and W. Wong, Nuclear Instruments and Methods in Physics Research Section A: Accelerators, Spectrometers, Detectors and Associated Equipment 581, 485 (2007).

[22] R. N. Wilke, M. Vassholz, and T. Salditt, Acta Crystallographica Section A 69, 490 (2013).

[23] B. L. Henke, E. M. Gullikson, and J. C. Davis, Atomic Data And Nuclear Data Tables 54, 181 (1993).

[24] B. McCallum and R. Bates, Journal of Modern Optics 36, 619 (1989).

[25] H. N. Chapman, A. Barty, S. Marchesini, A. Noy, S. P. Hau-Riege, C. Cui, M. R. Howells, R. Rosen, H. He, J. C. H. Spence, U. Weierstall, T. Beetz, C. Jacobsen, and D. Shapiro, J. Opt. Soc. Am. A 23, 1179 (2006).

[26] S. Flewett, H. M. Quiney, C. Q. Tran, and K. A. Nugent, Optics Letters 34, 2198 (2009). 